\title{
CONOCIMIENTO DE LAS ACCIONES DE PREVENCIÓN Y DENUNCIA DEL ACOSO SEXUAL ENTRE ESTUDIANTES DE TRABAJO SOCIAL DE UNA INSTITUCIÓN DE EDUCACIÓN SUPERIOR EN MÉXICO
}

\section{Knowledge of sexual harassment prevention and reporting actions among social work students at a higher education institution in Mexico}

\section{Luz Alejandra Escalera Silva}

Facultad de Trabajo Social y Desarrollo Humano de la

Universidad Autónoma de Nuevo León. México.

ORCID: 0000-0002-3214-2834

Correo-e: luz.escaleraslv@uanl.edu.mx

\section{Sandra Rubí Amador Corral}

Facultad de Trabajo Social y Desarrollo Humano de la

Universidad Autónoma de Nuevo León. México.

ORCID: 0000-0002-4814-6071

Correo-e. sandra.amadorcrr@uanl.edu.mx

\section{Recibido: 30/10/2020 • Aprobado: 15/12/2020}

Cómo citar: Escalera Silva, L. A., \& Amador Corral, S. R. (2021). Conocimiento de las acciones de prevención y denuncia del acoso sexual entre estudiantes de trabajo social de una institución de educación superior en México. Ciencia y Sociedad, 46(1), 9-22. https://doi.org/10.22206/cys.2021.v46i1.pp9-22

\section{Resumen}

Este estudio presenta una descripción de los diferentes mecanismos que se han empleado en las universidades públicas estatales en México para intervenir en casos de violencia de género. Su objetivo es analizar el conocimiento que las estudiantes de una escuela de educación superior tienen acerca de las acciones que su institución ha diseñado para prevenir, atender y erradicar el hostigamiento sexual en la comunidad universitaria. Se realizó un estudio descriptivo a través de la aplicación de una escala Likert a estudiantes mujeres de una escuela de educación superior en México. Entre los hallazgos más sobresalientes se encontraron que las estudiantes desconocen los mecanismos de denuncia de hostigamiento y destacan la importancia de la difusión de las políticas institucionales para la presentación de la queja, así como la trascendencia del papel que desempeña el docente tutor en el empoderamiento de las estudiantes para la presentación de la denuncia de hostigamiento.

Palabras clave: hostigamiento sexual; acoso sexual; violencia de género; mujeres.

\begin{abstract}
This study presents a brief description of the different mechanisms that have been used in state public universities in Mexico to intervene in cases of gender violence. Its objective is to analyze the knowledge that students of a higher education school have about the actions that their institution has designed to prevent, attend to and eradicate sexual harassment in the university community. A descriptive study was carried out by applying a Likert scale to women students at a higher education school in Nuevo León, Mexico. Among the most outstanding findings were that the students were unaware of the mechanisms for reporting harassment and the importance of disseminating institutional policies for the filing of complaints, as well as the importance of the role played by the tutor in empowering students to file complaints of harassment.
\end{abstract}

Keywords: sexual harassment; sexual molestation; gender violence; women. 


\section{Introducción}

La violencia de género refiere a actos perjudiciales realizados en contra de una persona en relación a las diferencias que la sociedad asigna a hombres y mujeres; dentro de una interpretación amplia de este tipo de violencia se puede incluir la que va dirigida a hombres y nińos. No obstante, tanto históricamente como en la actualidad, este término se utiliza como una forma de poner de manifiesto la vulnerabilidad de las mujeres y las niñas a padecer o sufrir actos o situaciones que las perjudiquen en todo su ciclo de vida (Fondo Internacional de Emergencia de las Naciones Unidas para la Infancia, 2020).

Según la Organización Mundial de la Salud (2020), la violencia contra la mujer refiere todo acto de violencia que resulte o pueda tener consecuencias físicas, sexuales o psicológicas, incluye acciones como las amenazas, la coacción o la privación arbitraria de la libertad que se producen en la vida pública y/o privada. La violencia de género es una de las problemáticas que más aquejan a la sociedad mexicana, estudios realizados por Echeverría, Paredes y Kantún (2017); Buquet, Cooper, Mingo, y Moreno (2013); Trigg y Wittenstrom (1996); Fineran y Bolen (2006) y Cortázar (2019) han demostrado que las más afectadas por la violencia de género son las mujeres.

Según los resultados de la Encuesta Nacional sobre la Dinámica de las Relaciones en los Hogares (ENDIREH) 2016, de una población encuestada de 46.5 millones, un $66 \%$ de mujeres de quince años y más han padecido algún tipo de violencia en ámbitos como el familiar, comunitario, laboral y escolar. Los resultados del mismo instrumento arrojaron que la violencia en el contexto escolar asciende a $25.3 \%$, donde el $10.9 \%$ de las mujeres mexicanas manifestaron que durante su etapa como estudiante sufrieron violencia sexual como acoso y hostigamiento.

Mingo y Moreno (2017) citan a Feltes (2012) quien reportó un estudio realizado en 35 universidades europeas, encontrando que al menos un $60.7 \%$ de los estudiantes encuestados habrían sufrido un episodio de hostigamiento sexual en su paso por la universidad.

Autores como Fineran y Bennet (1999) y Fineran y Bolen (2006) encontraron que los varones reproducen estas prácticas dos veces más que las mujeres, señalando que el género es un indicador importante para perpetuar o padecer hostigamiento sexual en las universidades.

Actualmente, en México se han tomado diferentes medidas legislativas con la intención de prevenir, atender y erradicar la violencia contra las mujeres, así, la Ley General de Acceso de las Mujeres a una Vida Libre de Violencia (2007) reconoce cinco tipos de violencia contra la mujer, a saber, violencia psicológica, violencia física, violencia sexual, violencia económica y violencia patrimonial. Asimismo, identifica los entornos familiar, comunitario, institucional y, por último, el laboral y escolar-docente, como los ámbitos donde dicha violencia se presenta. En lo que respecta al ámbito laboral y escolar-docente, la ley define el acoso como una forma de violencia que el agresor ejerce sobre la víctima, consistente en la conducta no deseada de naturaleza sexual, que hace que la víctima se sienta ofendida, humillada o intimidada y conduce a un estado de indefensión y de riesgo para la víctima, contrario al hostigamiento sexual, en el que sí existe el ejercicio de poder en una relación de subordinación real de la víctima frente al agresor en los ámbitos laboral o escolar y se expresa en conductas verbales, físicas o ambas, relacionadas con la sexualidad de connotación lasciva.

Los episodios de acoso y hostigamiento sexual cada vez más frecuentes han obligado a las instituciones a responsabilizarse por la seguridad de sus estudiantes y tomar medidas de control para la prevención, atención y sanción que garanticen a las estudiantes espacios educativos libres de violencia. 
En relación con lo anterior, a continuación, se presenta la revisión de las acciones que las universidades públicas estatales han tomado respecto al combate de la violencia de género, especialmente las acciones que la Universidad Autónoma de Nuevo León, nuestro escenario de estudio, ha realizado para erradicar, prevenir y atender casos de violencia de género dentro de sus instalaciones. Además, como objetivo general de este estudio se describe el conocimiento que las estudiantes la Facultad de Trabajo Social tienen sobre dichas acciones.

\section{Medidas para atender, sancionar y erradicar la violencia de género en las universidades públicas de México}

Actualmente, hemos sido testigos de una cantidad enorme de denuncias de estudiantes por hostigamiento sexual de parte de docentes universitarios, exigiendo a sus universidades tomen las medidas formales necesarias para garantizarles espacios educativos libres de violencia de género. Por esta razón nos dimos a la tarea de hacer una revisión de las acciones institucionales que las treinta y tres (33) universidades públicas estatales en México han diseñado para la atención de los casos de violencia de género que se sucedan. A continuación, se presenta una relación de las universidades que han adoptado protocolos de actuación en contra de la violencia de género.

Tabla 1. Relación de universidades con Protocolo de Acción para combatir violencia de género

\begin{tabular}{|c|c|c|c|}
\hline $\begin{array}{c}\text { Universidad pública } \\
\text { estatal }\end{array}$ & $\begin{array}{c}\text { Cuenta } \\
\text { con } \\
\text { protocolo }\end{array}$ & $\begin{array}{c}\text { Universidad } \\
\text { pública estatal }\end{array}$ & $\begin{array}{c}\text { Cuenta con } \\
\text { protocolo }\end{array}$ \\
\hline $\begin{array}{c}\text { Universidad Autónoma de } \\
\text { Aguascalientes }\end{array}$ & $\bullet$ & $\begin{array}{c}\text { Universidad } \\
\text { Autónoma } \\
\text { del Estado de } \\
\text { Morelos }\end{array}$ & $\bullet$ \\
\hline $\begin{array}{c}\text { Universidad Autónoma de } \\
\text { Baja California }\end{array}$ & $\bullet$ & $\begin{array}{c}\text { Universidad } \\
\text { Autónoma de } \\
\text { Nayarit }\end{array}$ & $\bullet$ \\
\hline $\begin{array}{c}\text { Universidad Autónoma de } \\
\text { Baja California Sur }\end{array}$ & $\bullet$ & $\begin{array}{c}\text { Universidad } \\
\text { Autónoma de } \\
\text { Nuevo León }\end{array}$ & $\bullet$ \\
\hline
\end{tabular}

\begin{tabular}{|c|c|c|c|}
\hline $\begin{array}{l}\text { Universidad Autónoma de } \\
\text { Campeche }\end{array}$ & & $\begin{array}{c}\text { Universidad } \\
\text { Autónoma } \\
\text { Benito Juárez de } \\
\text { Oaxaca }\end{array}$ & • \\
\hline $\begin{array}{l}\text { Universidad Autónoma de } \\
\text { Coahuila }\end{array}$ & & $\begin{array}{c}\text { Benemérita } \\
\text { Universidad } \\
\text { Autónoma de } \\
\text { Puebla }\end{array}$ & • \\
\hline Universidad de Colima & & $\begin{array}{c}\text { Universidad } \\
\text { Autónoma de } \\
\text { Querétaro }\end{array}$ & • \\
\hline $\begin{array}{l}\text { Universidad Autónoma de } \\
\text { Chiapas }\end{array}$ & $\bullet$ & $\begin{array}{l}\text { Universidad de } \\
\text { Quintana Roo }\end{array}$ & • \\
\hline $\begin{array}{l}\text { Universidad Autónoma de } \\
\text { Chihuahua }\end{array}$ & & $\begin{array}{l}\text { Universidad } \\
\text { Autónoma de } \\
\text { San Luis Potosí }\end{array}$ & $\bullet$ \\
\hline $\begin{array}{c}\text { Universidad Autónoma de } \\
\text { Ciudad Juárez }\end{array}$ & & $\begin{array}{c}\text { Universidad } \\
\text { Autónoma de } \\
\text { Sinaloa }\end{array}$ & $\bullet$ \\
\hline $\begin{array}{l}\text { Universidad Juárez del } \\
\text { Estado de Durango }\end{array}$ & $\bullet$ & $\begin{array}{l}\text { Universidad de } \\
\text { Sonora }\end{array}$ & \\
\hline $\begin{array}{l}\text { Universidad de } \\
\text { Guanajuato }\end{array}$ & & $\begin{array}{l}\text { Universidad } \\
\text { Juárez } \\
\text { Autónoma de } \\
\text { Tabasco }\end{array}$ & \\
\hline $\begin{array}{l}\text { Universidad Autónoma de } \\
\text { Guerrero }\end{array}$ & & $\begin{array}{l}\text { Universidad } \\
\text { Autónoma de } \\
\text { Tamaulipas }\end{array}$ & \\
\hline $\begin{array}{l}\text { Universidad Autónoma del } \\
\text { Estado de Hidalgo }\end{array}$ & $\bullet$ & $\begin{array}{c}\text { Universidad } \\
\text { Autónoma de } \\
\text { Tlaxcala }\end{array}$ & \\
\hline $\begin{array}{l}\text { Universidad de } \\
\text { Guadalajara }\end{array}$ & & $\begin{array}{l}\text { Universidad } \\
\text { Veracruzana }\end{array}$ & $\bullet$ \\
\hline $\begin{array}{l}\text { Universidad Autónoma del } \\
\text { Estado de México }\end{array}$ & & $\begin{array}{c}\text { Universidad } \\
\text { Autónoma de } \\
\text { Yucatán }\end{array}$ & \\
\hline $\begin{array}{l}\text { Universidad Michoacana } \\
\text { de San Nicolás de Hidalgo }\end{array}$ & & $\begin{array}{c}\text { Universidad } \\
\text { Autónoma de } \\
\text { Zacatecas }\end{array}$ & \\
\hline
\end{tabular}

Fuente: elaboración propia.

La Universidad de Aguascalientes cuenta con el Protocolo de Actuación ante situaciones de Acoso, Discriminación y Violencia desde el 2017, cuyo objetivo principal es dotar a la comunidad universitaria de medidas formales para atender situaciones de acoso, hostigamiento, discriminación y violencia que se presenten en espacios o ámbitos universitarios. Para cumplir con este objetivo, este protocolo contempla como única instancia competente para actuaciones en materia de acoso, hostigamiento, discriminación o violencia a la Defensoría de los Derechos Universitarios. 
En el caso de la Universidad Autónoma de Coahuila, el Protocolo de actuación para prevenir, atender, sancionar y erradicar la violencia de género al interior de la Universidad Autónoma de Coahuila, designa como autoridad competente para conocer sobre denuncias formales al Comité para Prevenir la Violencia de Género, ubicado en las instalaciones de las tres coordinaciones de unidad de la universidad, en Saltillo, Torreón y Monclova. El procedimiento de primera instancia comienza con la denuncia formal ante el Comité, luego se solicita la comparecencia de las partes ante el Comité, ya sea personalmente, por escrito o a través de un tutor o representante legal; se prosigue a la presentación de pruebas para que las partes sustenten sus casos. Una vez valoradas las pruebas, los miembros del comité emitirán un dictamen. Dicho dictamen es canalizado al Consejo Directivo que tendrá siete días para analizar el dictamen y emitir una resolución. En la segunda instancia, el dictamen es emitido por la Comisión de Honor Justicia del H. Consejo Universitario. Algo que destacar de este protocolo es que, a pesar de ser de observancia general para toda la comunidad universitaria, no contempla la figura del hostigamiento sexual.

En noviembre de 2019, se promueven los Lineamientos de la Unidad de Género de la Universidad Autónoma de Chihuahua, cuya observancia es obligatoria en toda la universidad y, a su vez, contempla las acciones institucionales para asegurar la igualdad entre hombres y mujeres en las dependencias universitarias. En estos lineamientos se establece que, en caso de denuncia formal por violencia de género en alguna dependencia universitaria, la Defensoría de Derechos Universitarios será el organismo competente para dictaminar los efectos legales a que haya dado lugar el incidente en términos de la normatividad aplicable al caso concreto.

La Universidad de Guanajuato cuenta con el equipo UGénero, un grupo interdisciplinario conformado por personal de la universidad, responsable de atender situaciones de violencia de género y aplicar el Protocolo de Atención a Casos de Violencia y Programa Institucional de Igualdad de Género. Un elemento importante de este protocolo es que es aplicable en casos en que la violencia se ejerza sobre una persona de la comunidad universitaria, o cuando son las autoridades universitarias las agresoras y dichas agresiones sean producto de vínculos generados en las relaciones asimétricas, reales o simbólicas derivadas de la realización de actividades académicas o laborales.

La Universidad Autónoma del Estado de México cuenta con el Protocolo de actuación para prevenir y eliminar la violencia y la discriminación contra la diversidad de la identidad sexual y de género, cuyo objetivo general es establecer un programa de actuación que se aplique de manera general en todos los espacios universitarios para asegurar el pleno desarrollo de la comunidad universitaria. Este protocolo faculta a la Defensoría de los Derechos Universitarios de la UAEM para conocer y resolver cualquier queja que implique una conducta violenta derivada de discriminación o por cuestión de género o diversidad sexual.

Asimismo, la Universidad Michoacana de San Nicolás de Hidalgo cuenta desde el 2016 con el Protocolo para la Prevención, Actuación y Erradicación de la Violencia de Género, su procedimiento de actuación implica medidas de prevención, detección, canalización, atención, acompañamiento y sanción para erradicar la violencia de género en la comunidad universitaria. La autoridad competente para conocer, atender y sancionar en caso de violencia de género es la Defensoría de Derechos Humanos Universitarios Nicolaítas.

Por su parte, la Universidad Autónoma de Morelos cuenta con el Protocolo de Actuación para la Prevención y Atención Temprana de Casos de Violencia en la UAEM cuyo objetivo principal es prevenir, atender y sancionar cuestiones derivadas de violencia 
de género como acoso sexual, hostigamiento sexual y conductas discriminatorias por razón de la orientación sexual, identidad o expresión de género, así como violencia por acoso laboral y violencia por acoso escolar. La instancia competente para conocer y resolver las quejas que afecten a la comunidad universitaria es la Unidad de Atención de Víctimas de Violencia.

La Universidad Autónoma Benito Juárez de Oaxaca tiene el Protocolo para la atención y erradicación de la discriminación, el hostigamiento, acoso sexual y la violencia en contra de las mujeres, cuyo propósito es la implementación de un procedimiento para prevención, atención y sanción de la violencia contra las mujeres, del hostigamiento y del acoso sexual en la universidad. La queja se presenta ante la Defensoría de Derechos Universitarios.

A su vez, la Benemérita Universidad Autónoma de Puebla implementó desde noviembre de 2019 el Protocolo para la Prevención y Atención de la Discriminación y la Violencia de Género, su objetivo general es establecer las instancias encargadas de prevenir, atender y sancionar la violencia de género en la comunidad universitaria, así como consolidar una cultura de perspectiva de género para garantizar un espacio universitario incluyente y libre de violencia. La Defensoría de los Derechos Universitarios es la instancia competente para conocer sobre conductas que vulneren los derechos de los estudiantes, de violencia de género o de conductas discriminatorias.

En la Universidad Autónoma de Querétaro (UAQ) se creó en 2019 el Protocolo de actuación e intervención en materia de Violencia de Género, su objetivo es garantizar espacios libres de violencia de género en la universidad, estableciendo los mecanismos necesarios para prevenir, atender, investigar, sancionar y erradicar la violencia y discriminación por motivo de género. La instancia responsable de la atención y solución de quejas es la Unidad de Atención de Violencia de Género (UAVIG), misma que se encuentra adscrita a la Oficina del Abogado General de la UAQ.

La Universidad de Quintana Roo (UQR) emplea el Procedimiento para la Atención de Casos de Hostigamiento y/o Acoso Sexual en Agravio del Alumnado de la UQR para resolver sobre los casos de hostigamiento y/o acoso sexual cometidos en la universidad o por algún integrante de la comunidad universitaria, la unidad responsable para conocer las quejas por acoso u hostigamiento es la Dirección General de Asuntos Jurídicos a través del Comité de Equidad y Género.

En agosto de 2017, se aprobó el Protocolo de Entrada para Prevenir, Atender, Intervenir, Sancionar y Erradicar el Acoso y el Hostigamiento Sexual en la Universidad Autónoma de San Luis Potosí, su finalidad es establecer los mecanismos para prevenir, atender, intervenir, sancionar y erradicar el acoso y el hostigamiento en la comunidad universitaria. La solicitud de procedimiento de acoso u hostigamiento sexual deberán presentarse ante la Defensoría de los Derechos Universitarios y hacerlas de conocimiento para su registro en la Dirección de Fortalecimiento Humano.

La Universidad Autónoma de Sinaloa cuenta con el Reglamento para la Prevención, Atención y sanción del hostigamiento y acoso sexual en la UAS en el que establece los lineamientos para la presentación y resolución de quejas que se presenten en el plantel, una característica importante de este documento es que divide las conductas de acoso y hostigamiento sexual en I. de gravedad leve, II. De gravedad media y III. De gravedad alta; este tipo de clasificaciones minimiza la ofensa hacia las víctimas, lejos de apoyar a la prevención y erradicación de la violencia sexual en la universidad, ya ningún tipo de violencia tendría que ser tolerada. 
Por su parte, la Universidad Veracruzana tiene la Guía para la atención de casos de hostigamiento y acoso sexual, la autoridad competente para conocer las denuncias es la Defensoría de los Derechos Universitarios que remitirá el caso a la Unidad de Género de la Universidad Veracruzana. En este procedimiento de atención de denuncia no figuran la conciliación o la mediación, ya que consideran que estos mecanismos de solución de conflictos tienden a revictimizar a las personas y las exponen a un mayor riesgo en caso de hostigamiento y acoso.

En el caso de la Universidad Autónoma de Nuevo León, nuestro contexto de estudio, el Protocolo de atención para casos de acoso u hostigamiento sexual entró en vigor a partir de marzo del 2019 y es aplicable a todo integrante de la comunidad universitaria en los supuesto de actos de acoso u hostigamiento sexual ocurridos en las instalaciones institucionales oficiales, cualquier instalación en donde se realice una actividad oficial de la universidad, así como en espacios distintos a los recintos institucionales, siempre y cuando intervenga una persona perteneciente a la comunidad universitaria y transgredan la normativa, el orden, la disciplina, los principios y valores que deben guiar la conducta de los universitarios afectando las relaciones académicas y laborales. La autoridad competente para conocer cualquier queja sobre acoso u hostigamiento sexual es la UNIIGÉNERO, que tendrá la facultad de iniciar el procedimiento de investigación que marca el Protocolo, siempre que exista una queja formal o se trate de acontecimientos que involucren a menores de edad, en cuyo caso se actuará de oficio.

Las fases para la implementación del protocolo son: I. La orientación. Etapa en la que la persona que considere haber sido víctima de un acto de acoso u hostigamiento sexual acude ante la UNIIGÉNERO para solicitar información sobre el procedimiento de queja; II. La queja formal. Etapa con la que comienza el procedimiento formal de acción del protocolo, que puede ser presentada de manera individual o colectiva ante la UNIIGÉNERO y puede ser por escrito, por comparecencia o por correo electrónico y le es asignado un número de expediente. Una vez que UNIIGÉNERO recibe la queja formal, tendrá diez días para determinar la procedencia de la queja; III. La Canalización a CIIGEN. En caso de determinarse la procedencia de la queja, esta se canalizará a la Comisión para la Investigación en Igualdad de Género (CIIGEN), y; IV. La Investigación y proceso interno. La CIIGEN recibe el expediente e inicia el proceso de investigación, la CIIGEN dará vista a las partes para que dentro de diez días manifieste por escrito lo que a su derecho convenga y ratificando debidamente su escrito. Realizadas las notificaciones por parte de la CIIGEN se citará a una audiencia en la Facultad de Derechos y Criminología para ofrecer pruebas. La CIIGEN deberá determinar en cinco días hábiles la resolución del caso.

Una peculiaridad de este protocolo es que solo permite que se presente la queja dentro de los 12 meses siguientes del episodio de acoso u hostigamiento, siendo el único protocolo de los antes mencionados, en señalar prescripción de la acción.

Como se ha podido constatar a través de la revisión de protocolos universitarios, las escuelas de educación superior han implementado diferentes mecanismos de acción para intervenir en contra de la violencia de género, en este contexto queda la interrogante, ¿Cuál es el conocimiento que las estudiantes tienen sobre las políticas de protección que sus instituciones han diseñado para asegurarles espacios libres de violencia? ¿Son estas medidas suficientes?

\section{Metodología}

Se realizó un estudio descriptivo. Para recabar la información se aplicó una escala de Likert $(1=$ de acuerdo, $2=$ medianamente de acuerdo, $3=$ en desacuerdo) $\mathrm{a}$ una muestra aleatoria de estudiantes exclusivamente mujeres de una población total de 1593 estudiantes de la Facultad de Trabajo Social de la Universidad 
Autónoma de Nuevo León. La muestra se calculó a partir del tamaño de la población y considerando un intervalo de confianza de 5 y un nivel de confiabilidad de $95 \%$, utilizando la fórmula estadística:

$$
\begin{gathered}
\frac{z^{2} \times p(1-p)}{e^{2}} \\
1+\frac{\left(z^{2} \times p(1-p)\right.}{e^{2} N}
\end{gathered}
$$

Obteniendo como resultado una muestra de 310 estudiantes. El trabajo de campo se realizó en el mes de mayo de 2020. Por motivos relacionados a la pandemia COVID-19 y siguiendo las indicaciones de las autoridades sanitarias de guardar distanciamiento social, con apoyo del Centro de Atención Integral del Estudiante de la facultad de Trabajo Social, se aplicó de manera virtual a través de la plataforma google forms, cuando se alcanzó el tamaño de la muestra deseada (310), se cerró el formulario en la plataforma. El cuestionario se redactó con base en el estudio de Hernández, Jiménez y Galicia (2015), adaptándolo a las características de la población de estudio, dejando como resultado 10 reactivos (puede consultarse en el anexo 1).

Al momento de contestar la encuesta se les hace saber a las estudiantes que la información que proporcionan es de carácter confidencial y sería utilizada para la realización de esta investigación. El análisis de la información obtenida se realizó utilizando el software estadístico SPSS versión 20.

\section{Resultados y discusión}

Las edades de las estudiantes encuestadas oscilan entre los 17 y los 25 años de edad, y se encuentran entre los semestres segundo y octavo de la licenciatura en Trabajo Social y Desarrollo Humano.

A las estudiantes se les cuestionó si sabían qué es el hostigamiento sexual, un $82.7 \%$ contestó estar de acuerdo; un $17.3 \%$ contestó no saber, lo que resulta un foco rojo para la seguridad de los estudiantes que, al no tener conocimiento de qué comportamientos constituyen hostigamiento sexual, guardan silencio y no se quejan formalmente ante sus universidades (DeSouza y Solberg, 2003).

Otro foco rojo que encontramos dentro del estudio que realizamos, fue que solo un $24.1 \%$ de las estudiantes de trabajo social encuestadas tienen claro cuál es el procedimiento formal para denunciar el hostigamiento sexual en su universidad. Un $53.9 \%$ contestó estar medianamente de acuerdo, lo que hace dudar que conozcan el procedimiento, y un $24.1 \%$ contestó no saber el procedimiento de denuncia. Para Weiss y Lasky (2017) la claridad y difusión en las políticas de atención de violencia sexual en la universidad constituye un elemento importante para reducir la situación de vulnerabilidad de las estudiantes. Es necesario encaminar acciones para visibilizar las políticas institucionales en contra de violencia de género.

Eventos de hostigamiento sexual pueden llegar a causar severas consecuencias psicológicas a corto y largo plazo entre las víctimas como ansiedad y depresión (Bondestam \& Lundqvist, 2020), la existencia de redes que apoyen a las víctimas a confrontar y sobrellevar sucesos trascendentes en sus vidas resulta de gran importancia para el acompañamiento de la víctima (Juárez, 2006; Hernández, et al., 2015), se le cuestionó a las estudiantes si han percibido la existencia de redes de apoyo, un $38.2 \%$ contestó que sí, sin embargo, un 50 \% mencionó estar medianamente de acuerdo y un $11.2 \%$ señaló no tener conocimiento de alguna red de apoyo. Gestionar espacios libres de violencia también requiere de la creación de redes a través de grupos de mujeres que acompañen y apoyen a la víctima en el procedimiento institucional de denuncia.

Otro elemento importante para evitar situaciones de hostigamiento sexual es el conocimiento de las 
vías de denuncia formal en caso de ser víctimas de hostigamiento (D’Enbeau, 2019), un $37.6 \%$ de las estudiantes encuestadas mencionaron saber cuáles son las vías de denuncia, mientras que un $37.6 \%$ contestó estar medianamente de acuerdo y un 24.8 $\%$ no sabe cuáles son las vías de denuncia.

Las campañas de difusión son fundamentales para la visibilización del problema de violencia contra la mujer, que permea en la sociedad mexicana. Con la finalidad de saber si la facultad contribuye a la sensibilización de la importancia de erradicar la violencia contra la mujer, se les preguntó a las estudiantes si especificamente su facultad hace énfasis en promover campañas en contra de la violencia contra las mujeres y el hostigamiento sexual, un $60.3 \%$ contestó estar en conocimiento de las campañas y un $45.9 \%$ señaló que estar en conocimiento de dichas campañas han sido determinantes para no ser víctimas. Además, se indagó entre las estudiantes si consideraban que los profesores en sus materias fomentaban el aprendizaje con relación al tema de la violencia contra las mujeres a lo que un $56.6 \%$ contestó reconocer el esfuerzo que los académicos de su facultad realizan en temas de violencia y hostigamiento sexual para visibilizar la violencia contra la mujer.

Para Weiss y Lasky (2017) la confianza que pueda generarse entre la autoridad académica y la víctima de hostigamiento sexual es un elemento fundamental para que la víctima se empodere y denuncie. Se les preguntó a las estudiantes si perciben la existencia de alguna autoridad de confianza para contar situaciones de hostigamiento en su facultad, a lo que un $65.3 \%$ contestó estar de acuerdo, un dato importante que se arrojó en torno a este punto fue el reconocimiento de la figura del tutor académico como autoridad de confianza entre las estudiantes.

Por último, se consideró importante saber si la comunidad estudiantil está en conocimiento de las sanciones que se aplican en los casos de hostiga- miento sexual, un $32.2 \%$ contestó saber cuáles son las posibles sanciones, pero un importante $47.5 \%$ de las estudiantes encuestadas señalaron estar medianamente de acuerdo y un $20.2 \%$ desconoce las posibles sanciones, datos que invitan a la comunidad académica a la reflexión sobre las acciones de difusión que se están tomando en la institución educativa.

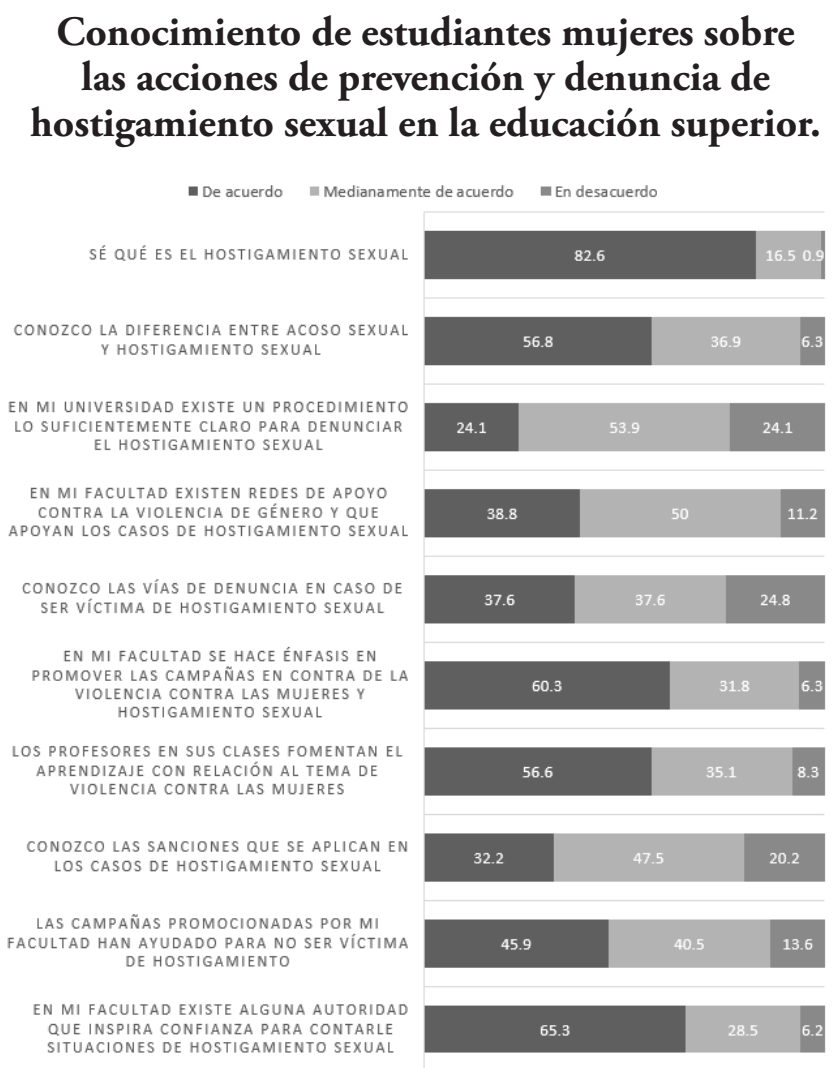

Figura 1. Resultados del estudio "Conocimiento de estudiantes mujeres sobre las acciones de prevención y denuncia de hostigamiento sexual en la educación superior"

Fuente: elaboración propia.

\section{Conclusiones}

La situación de desigualdad y violencia ha generado la creación de mecanismos de prevención, atención y sanción al acoso y al hostigamiento sexual. Sin embargo, es de trascendental importancia tener el conocimiento de si se cumple con el objetivo de los 
mecanismos creados, es decir, si las personas receptoras de los mecanismos de atención, se sienten seguras y conformes con las políticas que se tienen para atender los casos de violencia en la Institución Educativa.

Los resultados encontrados ponen en evidencia la necesidad de diseñar un plan para promover el Protocolo de atención para casos de acoso y hostigamiento sexual UANL en la comunidad universitaria y garantizar que todas las estudiantes conozcan el procedimiento de denuncia en la Universidad Autónoma de Nuevo León.

Asimismo, la difusión de las vías de denuncia entre las estudiantes y la creación de redes de apoyo que acompañen a la víctima durante el procedimiento de denuncia, son determinantes para fortalecer la solidaridad y empatía entre la población universitaria y lograr la erradicación de conductas de hostigamiento sexual en la universidad.

Las campañas para evitar la violencia contra la mujer empleada en la Facultad de Trabajo Social y Desarrollo Humano de la Universidad Autónoma de Nuevo León, así como la promoción del tema de la violencia contra las mujeres por parte de los maestros de la institución, han sido efectivas para visibilizar y sensibilizar a las estudiantes sobre el tema.

La confianza generada entre la autoridad académica y la víctima de hostigamiento sexual es un elemento importante para el proceso de denuncia, en este contexto, los resultados encontrados señalan que el programa de tutorías ha representado un papel fundamental como generador de confianza y puente de comunicación entre la institución y la estudiante.

Es importante llevar a cabo acciones de difusión respecto a las sanciones que pueden resultar de este procedimiento, ya que los mecanismos de acción y sus consecuencias no están llegando de manera eficaz a la comunidad universitaria. Buscar que la totalidad de nuestros alumnos conozcan qué es la violencia, el acoso y el hostigamiento, y consecuentemente tener una población educativa informada y capaz de detectar cualquier vulnerabilidad a sus derechos.

\section{Referencias}

Benemerita Universidad Autónoma de Puebla. (20 de mayo de 2020). Procedimiento para la atención de denuncias sobre discriminación, hostigamiento y acoso sexual en la BUAP. Recuperado de https:/genero.buap.mx/sites/default/files/ protocolo\%20completo.pdf

Bondestam, F., \& Lundqvist, M. (2020). Sexual harassment in higher education - a systemic review. European Journal of Higher Education, 2-24. doi:https://doi.org/10.1080/21568235. 2020.1729833

Buquet, A., Cooper, J., Mingo, A., \& Moreno, H. (2013). Intrusas en la Universidad. México: UNAM.

Cámara de Diputados del H. Congreso de la Unión. (2007). Ley General de Acceso de las Mujeres a una Vida Libre de Violencia. México.

Cortázar, F. (2019). Acoso y hostigamiento de género en la Universidad de Guadalajara. Habla el estudiantado. La Ventana. Revista de estudios de género, 175-204.

D'Enbeau, S. (2019). Communicating policy-practice orientations: the case of sexual violence response among college students. Journal of Applied Communication Research, 420-438.

DeSouza, E., \& Solberg, J. (2003). Incidence and dimensions of sexual harassment across cultures. En M. Paludi, \& C. Paludi, Academic and Workplace Sexual Harassment: A Handbook of Cultural, Social Science, Management, and Legal Perspectives (págs. 4-30). Westport: Praegrer.

Echeverría, R., Paredes, L., \& Kantún, C. (2017). Acoso y hostigamiento sexual en estudiantes universitarios: un acercamiento cuantitativo. Enseñanza de investigación en psicología, 15-26. 
Fineran, S., \& Bennet, L. (1999). Gender and power issues of peer sexual harassment. Journal of Interpersonal Violence, 626-641.

Finerarn, S., \& Bolen, R. (2006). Risk Factors for Peer Sexual Harassment in Schools. Journal of Interpersonal Violence, 1169-1190.

Fondo Internacional de Emergencia de las Naciones Unidas para la Infancia. (2020). La violencia de género en situaciones de emergencia. ONU. Recuperado de https://www.unicef.org/spanish/ protection/57929_58001.html

Hernández, C., Jiménez, M., \& Galicia , S. (2015). La Percepción de las Mujeres hacia las Acciones de Prevención, Denuncia y Erradicación del Hostigamiento Sexual en una Escuela del Instituto Politécnico Nacional en México. Fomación Universitaria, 66-74.

Hernández, C., Jiménez, M., \& Guadarrama, E. (2015). La percepción del hostigamiento y acoso sexual en mujeres estudiantes de dos instituciones de educación superior. Revista Educación Superior, 63-82.

INEGI. (2016). Encuesta Nacional sobre la Dinámica de las Relaciones en los Hogares (ENDIREH). México: INEGI.

Juárez, C. (2006). Ya no quisiera ni ser yo: La experiencia de la violencia doméstica en un grupo de mujeres y varones provenientes de zonas rurales y urbanas en México. Cataluña: Universitat Rovira i Virgili. Departament d'Antropologia, Filosofia i Treball Social.

Mingo, A. (2013). Cuatro grados bajo cero. Mujeres en la universidad. En C. Agoff, I. Casique, \& R. Castro, Visibles en todas partes. Estudios sobre violencia contra las mujeres en multiples ámbitos (pp. 1033-118). México: Porrúa-UNAM.

Mingo, A., \& Moreno, H. (2017). Sexismo en la universidad. Estudios sociológicos, 571-596.

Organización Mundial de la Salud. (2020). Temas de Salud. Violencia contra la mujer. WHO. Obtenido de https://www.who.int/topics/gender_ based_violence/es/
Perkins, W., \& Warner, J. (2017). Sexual Violence Response and Prevention: Studies of Campus Policies and Practices. Journal of School Violence, 237-242.

Sipe, S., Douglas, C., \& Fisher, D. (2009). University students' perceptions of sexual harassment in the workplace. Equal Opportunities International, 336-350.

Trigg, M., \& Witternstrom, K. (1996). That's the way the world goes: Sexual harassment and New Jersey teenagers. Initiatives, 55-65.

Universidad Autónoma Benito Juárez de Oaxaca. (21 de 04 de 2020). Protocolo para la prevención, atención y erradicación de la discriminación, la violencia contra las mujeres, el hostigamiento y acoso sexual en la UABJO. Recuperado de http:// www.transparencia.uabjo.mx/obligaciones/ uabjo/articulo-70/fraccion-1/70-1-2219-protocolo-para-la-prevencion-atencion-y-erradicaci...-2020.pdf

Universidad Autónoma de Aguascalientes. (14 de marzo de 2020). Procotolo de actuación ante situaciones de acoso, discriminación y violencia. Recuperado de https://www.uaa.mx/eventos/ diciembre/CORREO_UNIVERSITARIO_ No3.pdf

Universidad Autónoma de Chihuahua. (14 de 03 de 2020). Lineamientos de la Unidad De Género de la Universidad Autónoma de Chibuahua. Recuperado de http://transparencia.uach.mx/informacion_publica_de_ oficio/fraccion_i/LINEAMIENTOS $\% 20$ DE\%20LA\%20UNIDAD\%20DE\%20 G\%C3\%89NERO\%20DE\%20LA\%20 UACH_F.pdf

Universidad Autónoma de Querétaro. (14 de mayo de 2020). Protocolo de Actuación e Intervención en Materia de Violencia de Género. Recuperado de https://www.uaq.mx/leyes/PROTOCOLO_29AGO.pdf 
Universidad Autonoma del Estado de Coahuila. (14 de 03 de 2020). Protocolo de actuación para prevenir, atender, sancionar y erradicar la violencia de género al interior de la Universidad Autónoma de Coahuila. Recuperado de http:// www.transparencia.uadec. $\mathrm{mx} /$ sassit/docs/ ProtocoloPASEVG.pdf

Universidad Autónoma del Estado de México. (15 de mayo de 2020). Protocolo de actuación para prevenir y eliminar la violencia y la discriminación contra la diversidad de la identidad sexual $y$ de género. Recuperado de http://defensoria. uaemex.mx/images/alertateyactua/acuerdo_ protocolo.pdf

Universidad Autónoma del Estado de Morelos. (14 de mayo de 2019). Protocolo de actuación para la prevención y atención temprana de casos de violencia en la UAEM. Obtenido de https:// www.uaem.mx/estudiantes-y-egresados/ procuraduria-de-los-derechos-academicos/ files/protocolo-prevencion-atencion-violencia.pdf

Universidad de Guanajuato. (14 de marzo de 2020). Protocolo de Atención a Casos de Violencia de Género en la Universidad de Guanajuato. Recuperado de UGénero: https://www.ugto.mx/ ugenero/images/pdf/acuerdo-protocolo-atencion-casos-de-violencia-ug-ugto.pdf
Universidad de Quintana Roo. (13 de septiembre de 2019). Procedimiento para la Atención de Casos de Hostigamiento y/o Acoso Sexual en Agravio del Alumnado de la Universidad de Quintana Roo. Recuperado dehttp://www.uqroo.mx/modelo_ equidad_genero/archivos/ACA-11-PR-06\%20 acoso\%20sexual\%20sep\%202019.pdf

Universidad de Veracruz. (20 de mayo de 2020). Guia para la atención de casos de hostigamiento y acoso sexual. Recuperado de https://www. uv.mx/coatza/admon/files/2015/11/guia.pdf Universidad Michoacana de San Nicolás de Hidalgo. (20 de mayo de 2019). Protocolo para la Prevención, Actuación y Erradicación de la Violencia de Género. Recuperado de http://www.colegio. umich.mx/assets/files/Protocolo\%20para\%20 la $\% 20$ prevencion $\% 20$ actuacion $\% 20 \mathrm{y} \% 20$ erradicacion $\% 20 \mathrm{de} \% 20 \mathrm{la} \% 20$ violencia $\% 20$ de\%20genero\%20en\%20la\%20UMSNH.pdf Valadez, A., \& Ríos, L. (2014). Percepciones de acoso y hostigamiento sexual contra las mujeres. Un estudio exploratorio. Revista electronica de Psicología Iztacala, 624-645.

Weiss, K., \& Lasky, N. (2017). Mandatory reporting of sexual misconduct at collage: A critical perspective. Journal of School Violence, 276-286. 


\section{Anexo 1}

\section{Instrumento:}

Conocimiento de las estudiantes de trabajo social hacia las acciones de prevención y denuncia del hostigamiento sexual en la educación superior.

La información proporcionada es de carácter confidencial y será utilizada para la realización de una investigación. Muchas gracias por tu colaboración.

\section{Edad.}

II. Semestre.

1. Sé qué es el hostigamiento sexual.

2. Conozco la diferencia entre acoso sexual y hostigamiento sexual.

3. En mi universidad existe un procedimiento lo suficientemente claro para denunciar el hostigamiento sexual.

4. En la facultad existen redes de apoyo contra la violencia de género y que apoyan los casos de hostigamiento sexual.

5. Conozco las vías de denuncia en caso de ser víctima de hostigamiento sexual.

6. En mi facultad se hace énfasis en promover las campañas en contra de la violencia contra las mujeres y hostigamiento sexual.

7. Los profesores en sus clases fomentan el aprendizaje con relación al tema de violencia contra las mujeres.

8. Conozco las sanciones que se aplican en los casos de hostigamiento sexual.

9. Las campañas promocionadas por mi facultad han ayudado para no ser víctima de hostigamiento.

10. En mi facultad existe alguna autoridad que inspira confianza para contarle situaciones de hostigamiento sexual. *Si tu respuesta fue sí, por favor señala cuál es el cargo que esta autoridad ostenta en tu facultad. 


\section{Anexo 2}

\section{Universidades públicas que cuentan con protocolos de acción para atender quejas en materia de violencia de género}

Tabla 2. Relación de protocolos universitarios

\begin{tabular}{|c|c|c|}
\hline Universidad pública estatal & Año de expedición & Protocolo \\
\hline Universidad Autónoma de Aguascalientes & 2017 & $\begin{array}{l}\text { Protocolo De Actuación Ante situaciones de Acoso, } \\
\text { Discriminación y Violencia }\end{array}$ \\
\hline Universidad Autónoma de Baja California & ---- & SIN PROTOCOLO 1 \\
\hline $\begin{array}{l}\text { Universidad Autónoma de Baja California } \\
\text { Sur }\end{array}$ & --- & SIN PROTOCOLO \\
\hline Universidad Autónoma de Campeche & ---- & SIN PROTOCOLO \\
\hline Universidad Autónoma de Coahuila & 2019 & $\begin{array}{l}\text { Protocolo de actuación para prevenir, atender, } \\
\text { sancionar y erradicar la violencia de género al interior } \\
\text { de la Universidad Autónoma de Coahuila }\end{array}$ \\
\hline Universidad de Colima & ---- & SIN PROTOCOLO \\
\hline Universidad Autónoma de Chiapas & $-\ldots-$ & SIN PROTOCOLO \\
\hline Universidad Autónoma de Chihuahua & 2019 & $\begin{array}{l}\text { Lineamientos Generales para la Igualdad de Género en } \\
\text { la Universidad Autónoma de Chihuahua }\end{array}$ \\
\hline Universidad Autónoma de Ciudad Juárez & ---- & SIN PROTOCOLO \\
\hline Universidad Juárez del Estado de Durango & ---- & SIN PROTOCOLO \\
\hline Universidad de Guanajuato & 2017 & $\begin{array}{l}\text { Protocolo de atención a casos de violencia y Programa } \\
\text { Institucional de igualdad de Género }\end{array}$ \\
\hline Universidad Autónoma de Guerrero & ---- & SIN PROTOCOLO \\
\hline $\begin{array}{l}\text { Universidad Autónoma del Estado de } \\
\text { Hidalgo }\end{array}$ & ---- & SIN PROTOCOLO \\
\hline Universidad de Guadalajara & ---- & SIN PROTOCOLO \\
\hline $\begin{array}{l}\text { Universidad Autónoma del Estado de } \\
\text { México }\end{array}$ & 2016 & $\begin{array}{l}\text { Protocolo de actuación para prevenir y eliminar la } \\
\text { violencia y la discriminación contra la diversidad de la } \\
\text { identidad sexual y de género }\end{array}$ \\
\hline $\begin{array}{l}\text { Universidad Michoacana de San Nicolás de } \\
\text { Hidalgo }\end{array}$ & 2016 & $\begin{array}{l}\text { Protocolo para la Prevención, Actuación y } \\
\text { Erradicación de la Violencia de Género }\end{array}$ \\
\hline $\begin{array}{l}\text { Universidad Autónoma del Estado de } \\
\text { Morelos }\end{array}$ & 2018 & $\begin{array}{l}\text { Protocolo de actuación para la prevención y atención } \\
\text { temprana de casos de violencia en la UAEM }\end{array}$ \\
\hline Universidad Autónoma de Nayarit & --- & SIN PROTOCOLO \\
\hline Universidad Autónoma de Nuevo León & 2016 & $\begin{array}{l}\text { Protocolo de atención para casos de acoso y } \\
\text { hostigamiento sexual en la UANL }\end{array}$ \\
\hline $\begin{array}{l}\text { Universidad Autónoma Benito Juárez de } \\
\text { Oaxaca }\end{array}$ & 2018 & $\begin{array}{l}\text { Protocolo para la prevención, atención y erradicación } \\
\text { de la discriminación, la violencia contra las mujeres, el } \\
\text { hostigamiento y acoso sexual en la UABJO }\end{array}$ \\
\hline
\end{tabular}

1. Se encontraron notas periodísticas que indican que solo la Facultad de Medicina y Psicología de la Universidad Autónoma de Baja California cuenta con un protocolo para atención a casos de acoso y hostigamiento sexual, sin embargo, no se localizó el documento en las páginas web. 
Luz Alejandra Escalera Silva y Sandra Rubí Amador Corral

\begin{tabular}{|l|c|l|}
\hline $\begin{array}{l}\text { Benemérita Universidad Autónoma de } \\
\text { Puebla }\end{array}$ & 2019 & $\begin{array}{l}\text { Protocolo para la prevención y atención de la } \\
\text { discriminación y la violencia de Género. }\end{array}$ \\
\hline Universidad Autónoma de Querétaro & 2018 & $\begin{array}{l}\text { Protocolo de Actuación e Intervención en materia de } \\
\text { violencia de género }\end{array}$ \\
\hline Universidad de Quintana Roo & 2018 & $\begin{array}{l}\text { Procedimiento para la atención de quejas contra } \\
\text { discriminación, acoso y hostigamiento sexual o laboral }\end{array}$ \\
\hline Universidad Autónoma de San Luis Potosí & 2017 & $\begin{array}{l}\text { Protocolo de entrada para prevenir, atender, intervenir, } \\
\text { sancionar y erradicar el acoso y el hostigamiento sexual } \\
\text { en la UASLP }\end{array}$ \\
\hline Universidad Autónoma de Sinaloa & 2012 & $\begin{array}{l}\text { Reglamento para la Prevención, Atención y sanción } \\
\text { del hostigamiento y acoso sexual en la UAS }\end{array}$ \\
\hline Universidad de Sonora & ---- & SIN PROTOCOLO \\
\hline Universidad Juárez Autónoma de Tabasco & ---- & SIN PROTOCOLO \\
\hline Universidad Autónoma de Tamaulipas & ---- & SIN PROTOCOLO \\
\hline Universidad Autónoma de Tlaxcala & ---- & SIN PROTOCOLO \\
\hline Universidad Veracruzana & 2014 & $\begin{array}{l}\text { Guía para la atención de casos de hostigamiento y } \\
\text { acoso sexual }\end{array}$ \\
\hline Universidad Autónoma de Yucatán & ---- & SIN PROTOCOLO \\
\hline Universidad Autónoma de Zacatecas & ---- & SIN PROTOCOLO \\
\hline
\end{tabular}

Fuente: elaboración propia. 